LCRL-JC-119501

PREPRINT

\title{
"Simulation of the Consistent Boltzmann Equation for Hard \\ Spheres and its Extension to Higher Densities"
}

\section{Francis J. Alexander, Alejandro L. Garcia and Berni J. Alder Institute for Scientific Computing Research Lawrence Livermore National Laboratory Livermore, $\mathrm{CA}$}

This paper was prepared for submission to XIII Sitges Conference, Barcelona, Spain, June, 1994

October, 1994

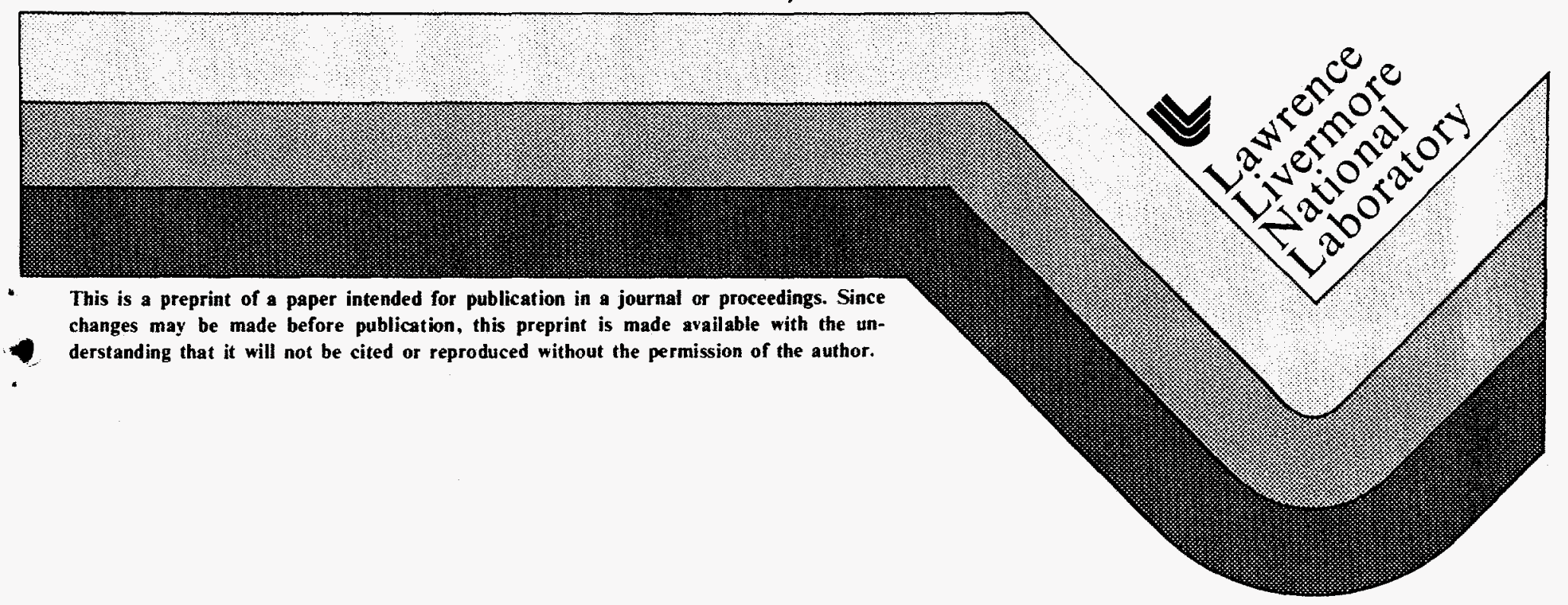




\section{DISCLAIMER}

This document was prepared as an account of work sponsored by an agency of the United States Government. Neither the United States Government nor the University of California nor any of their employees, makes any warranty, express or implied, or assumes any legal liability or responsibility for the accuracy, completeness, or usefulness of any information, apparatus, product, or process disclosed, or represents that its use would not infringe privately owned rights. Reference herein to any specific commercial product, process, or service by trade name, trademark, manufacturer, or otherwise, does not necessarily constitute or imply its endorsement, recommendation, or favoring by the United States Government or the University of California. The views and opinions of authors expressed herein do not necessarily state or reflect those of the United States Government or the University of California, and shall not be used for advertising or product endorsement purposes. 


\section{DISCLAIMER}

Portions of this document may be illegible in electronic image products. Images are produced from the best available original document. 


\title{
SIMULATION OF THE CONSISTENT BOLTZMANN EQUATION FOR HARD SPHERES AND ITS EXTENSION TO HIGHER DENSITIES
}

\author{
Francis J. Alexander, Alejandro L. Garcia*and Berni J. Alder \\ Institute for Scientific Computing Research L-416 \\ Lawrence Livermore National Laboratory \\ Livermore, California 94550
}

The direct simulation Monte Carlo method is modified with a post-collision displacement in order to obtain the hard sphere equation of state. This leads to consistent thermodynamic and transport properties in the low density regime. At higher densities, when the enhanced collision rate according to kinetic theory is introduced, the exact hard sphere equation of state is recovered, and the transport coefficients are comparable to those of the Enskog theory. The computational advantages of this scheme over hard sphere molecular dynamics are that it is significantly faster at low and moderate densities and that it is readily parallelizable.

\section{Introduction}

The direct simulation Monte Carlo (DSMC) method is a particle-based, numerical scheme for solving the nonlinear Boltzmann equation $[1,2,3]$. Rather than exactly calculating successive hard sphere (HS) collisions, as in molecular dynamics (MD) [4], DSMC generates collisions

*Permanent Address: Department of Physics, San Jose State University, San Jose, CA 95192-0106. 
stochastically with scattering rates and post-collision velocity distributions determined from the kinetic theory of a dilute gas. DSMC encounters the usual inconsistency of the Boltzmann equation, namely, it yields the transport properties for a dilute gas of hard spheres of diameter $\sigma$, yet results in an ideal gas equation of state (implying $\sigma=0$ ) [5]. In this paper, a modification to DSMC is introduced which removes this inconsistency and, in fact, recovers the exact HS equation of state at all densities.

The DSMC method solves the Boltzmann equation by using a representative random sample drawn from the actual velocity distribution. In the simulation, the state of the system is given by the positions and velocities of particles, $\left\{\vec{r}_{i}, \vec{v}_{i}\right\}$. The system evolves in two steps, advection (or free streaming) and collision. In free streaming, particles are propagated for a time $\Delta t$ as if they did not interact. In other words, their positions are updated to $\vec{r}_{i}+\vec{v}_{i} \Delta t$. Any particles that reach a boundary are reflected according to the boundary condition (e.g., specularly or diffusely).

After the advection step, the particles are sorted into cells to evaluate the collisions in the gas. Particles within a cell are randomly selected as collision partners according to the collision probabilities derived from dilute hard sphere kinetic theory. Conservation of momentum and energy provide four of the six equations needed to determine the post-collision velocities. The remaining two conditions are selected stochastically with the assumption that the direction of the post-collision relative velocity is uniformly distributed on the unit sphere. The spatial "coarse-graining" of particles into cells allows two particles to collide by simply being located within the same cell. Since only the magnitude of the relative velocity between particles is used in determining their collision probability, even particles that are moving away from each other may collide.

The DSMC scheme is only accurate when the time step is a fraction of the mean collision time and the cell volume is a fraction of a cubic mean free path. Because each particle in the simulation represents an effective number of molecules in the physical system, macroscopic systems may be accurately modeled by using as few as $10^{4}-10^{5}$ particles, with at least 20 particles per cubic mean free path [6]. A more detailed description of the standard DSNC method may be found in References [1] and [2].

The DSMC method was developed for use in rarefied gas dynamics to compute flows at 
high Knudsen number (ratio of mean free path to characteristic length) [7]. The algorithm has been thoroughly tested over the past 20 years and found to be in excellent agreement with both experimental data $[8,9]$ and molecular dynamics computations $[10,11]$. Recently, it was proved that DSMC is equivalent to a Monte Carlo solution of an equation "close" to the Boltzmann equation [3]. The DSMC method has also been useful in the study of nonequilibrium fluctuations [12], chemically reacting systems $[13,14]$ and nanoscale hydrodynamics [15].

\section{Non-ideal Gas DSMC}

To obtain a consistent equation of state, DSMC must be modified in the collision step to

include the extra separation, $\vec{d}(|\vec{d}|=\sigma)$, that the particles would have experienced if they had collided as hard spheres. Consider for simplicity a one-dimensional system with two hard rods of length $\sigma$ initially traveling toward each other. They collide when their centers are a distance $\sigma$ apart. After the collision, the distance between centers will be larger than the separation between similarly colliding point particles by a distance $2 \sigma[16]$. For hard spheres in three dimensions this effect generalizes to a displacement, $\vec{d}$ :

$$
\vec{d}=\frac{\left(\vec{v}_{1}^{\prime}-\vec{v}_{2}^{\prime}\right)-\left(\vec{v}_{1}-\vec{v}_{2}\right)}{\left|\left(\vec{v}_{1}^{\prime}-\vec{v}_{2}^{\prime}\right)-\left(\vec{v}_{1}-\vec{v}_{2}\right)\right|} \sigma=\frac{\vec{v}_{r}^{\prime}-\vec{v}_{r}}{\left|\vec{v}_{r}^{\prime}-\vec{v}_{r}\right|} \sigma,
$$

where the incoming velocities of the colliding particles 1 and 2 are $\vec{v}_{1}$ and $\vec{v}_{2}$, and the postcollisional velocities are $\vec{v}_{1}^{\prime}$ and $\vec{v}_{2}$ respectively; $\vec{v}_{r}$ is the relative velocity. Thus, particle 1 is displaced by the vector distance $\vec{d}$ and particle 2 by $-\vec{d}$. See Figure 1 for an example. In the low density limit the displacement yields the correct second virial coefficient. The average projection of the velocity change onto the line connecting centers of colliding particles after displacement, $\left\langle\vec{r}_{i j} \cdot \Delta \vec{v}_{i}\right\rangle$, the virial, resulting from this procedure is that of hard spheres at all densities.

\section{Dense Gas DSMC}

If, in addition to the displacement, $\vec{d}$, the Boltzmann collision rate is scaled by the so-called $Y$-factor, the enhanced probability of a collision due to the volume occupied by the spheres, a model in the spirit of Enskog results [18]. This density dependent $Y$ factor can be obtained 
from the HS equation state as determined by Monte Carlo and MD simulations and expressed in the Padé form [17]

$$
Y(n)=\frac{1+0.05556782 b_{2} n+0.01394451 b_{2}^{2} n^{2}-0.0013396 b_{2}^{3} n^{3}}{1-0.56943218 b_{2} n+0.08289011 b_{2}^{2} n^{2}}
$$

where $b_{2}=(2 / 3) \pi \sigma^{3}$ is the HS second virial coefficient. Collisions within a cell are generated with a rate $\Lambda\left(n^{*}\right)=Y\left(n^{*}\right) \Lambda_{00}\left(n^{*}\right)$, where $n^{*}=n \sigma^{3}$ is the reduced particle number density and $\Lambda_{00}$ is the Boltzmann collision rate:

$$
\Lambda_{00}(n)=2 N_{c} n \sigma^{2} \sqrt{\pi k_{B} T / m}
$$

In this expression $k_{B}$ is the Boltzmann constant, and $T$ is the temperature, $m$ is the particle mass and $N_{c}$ is the number of particles in a given cell. In the Enskog approximation, the mean free path for a dense gas is $\lambda=1 /\left(\sqrt{2} \pi n Y(n) \sigma^{2}\right)$ [18].

\section{Computer Simulations}

A series of computer simulations tested this model with the units determined by setting $m=1$, $\sigma=1$, and $k_{B} T=1$. The equilibrium pressure as a function of density can be determined from the virial and also by measuring the normal momentum transfer across a plane. Both procedures yield the HS equation of state within $1 \%$ for all densities (see Fig. 2) when the time step is less than 0.03 mean collision times. From the hydrodynamic expression for the direct scattering function, $S(k, \omega),[19]$, the sound speed obtained from the location of the Brillouin peak is in agreement with HS MD at low densities. At the higher densities, the Rayleigh and Brillouin peaks are not well separated, and accurate measurements of the sound speed cannot be made in this way. Furthermore, the radial distribution (pair correlation) function is that of a perfect gas so that the compressibility, as determined from the density fluctuations in a volume $V, \chi T=\left\langle\delta n^{2}\right\rangle V / k_{B} T n^{2}$ is that of a perfect gas and does not agree with $\chi_{T}=(\partial \log n / \partial p)_{T}$ as obtained directly from the equation of state.

The self-diffusion coefficient, $D$, is measured using the Einstein relation,

$$
D=\frac{1}{6 t}\left\langle\frac{1}{N} \sum_{i}^{N}\left(\vec{r}_{i}(t)-\vec{r}_{i}(0)\right)^{2}\right\rangle,
$$


where $N$ is the number of particles in the system and $t$ is the (long) time over which averages are taken. For densities up to $n^{*} \approx 0.3$ there is good agreement (within $5 \%$ ) with hard sphere MD and the Enskog self-diffusion prediction. However, at higher densities, the agreement fails because the post-collisional particle displacement $\vec{d}$ is of similar or larger magnitude than the mean free path. Also, as mentioned above, structural effects found at high densities in hard spheres are absent in this model and the backscattering events at these densities are not reproduced (i.e., there is no "caging").

The shear viscosity was measured by both equilibrium (Einstein relation and transverse current correlation function) and nonequilibrium (Poiseuille flow and relaxing velocity sine waves) techniques. For the thermal conductivity only the Einstein relation was used. The transport coefficients as functions of density are shown in Figures 3 and 4. For the shear viscosity, there is good agreement with both Enskog theory and HS MD at lower densities. At higher densities the measured shear viscosity shows better agreement with HS MD than does Enskog theory.

Poiseuille flows for various densities were generated in a channel by applying a constant external force on the particles parallel to the walls. At the walls, a thermal boundary condition was used; that is, particles colliding with a wall were emitted with a biased Maxwellian distribution at temperature $T$. The resulting velocity profile (See Figure 5) was fit assuming a parabolic form,

$$
U(x)=\left(\frac{n F}{2 \eta}\right)\left((L / 2)^{2}-x^{2}\right)+U_{s l i p}
$$

where $U_{\text {slip }}$ is the slip velocity at the walls, $F$ is the force applied to the fluid, and $L$ is the channel width. As can be seen in Fig 3, the viscosity obtained in this way agrees with alternative methods.

The Einstein relation allows one to assess the separate contributions to the transport coefficients. In HS MD there are two ways to transfer momentum and energy, namely by streaming and collisions. The former, the kinetic transport, is due to the motion of the particle. while potential transport consists of momentum and energy being instantaneously transferred in a collision from the center of one sphere to the center of its collision partner. The shear viscosity and thermal conductivity may then be decomposed into three distinct parts: the kinetic: 
potential, and cross contributions [20]. These separate terms can also be determined from the Enskog theory of hard spheres [18].

In the model presented in this paper the kinetic contribution to the fluxes is the same as that for uncorrelated hard spheres as given by the Enskog theory. The collisional transport, however, has two parts: exchange between colliding particles (which are in the same cell) and post-collision displacement. The viscosity, for example, then has the form

$$
\eta=\frac{m^{2}}{2 V k_{B} T t}\left\langle\left[\int_{0}^{t} \sum_{i}^{N} v_{x i}(s) v_{y i}(s) d s+\sum_{\text {coll.pairs }}\left(v_{x i}^{\prime}-v_{x i}\right) y_{i j}+\sum_{\text {coll.pairs }}\left(v_{x i}^{\prime} d_{y}-v_{x j}^{\prime} d_{y}\right)\right]^{2}\right\rangle,
$$

where $y_{i j}$ is the $y$-component of the distance between colliding particles $i$ and $j$ and $d_{y}$ is the

$y$-component of $\vec{d}$. The first term accounts for the kinetic transport; the second term for the transfer of momentum over the distance separating colliding particles $i$ and $j$, and the last term for the post-collision displacement. The second term in (5) corresponds to collisional momentum and energy transfer on a length scale on the order of a cell size. In both standard DSMC and its dense gas extension, the transport coefficients depend (weakly) on cell size $\Delta y$, yet this effect is small when $\Delta y$ is less than the mean free path [21]. In the limit of cell size tending to zero, this "grid error" vanishes (since $y_{i j} \rightarrow 0$ ). For all cases shown in Figure 3 , the grid error was within the error bars of the measured transport coefficients.

Good agreement with Enskog theory is found for the kinetic and cross terms of the shear viscosity and thermal conductivity at all densities [22]. The potential term in the shear viscosity is about twice that predicted by the Enskog theory; for thermal conductivity the potential term was about $25 \%$ larger than the Enskog predicted value. A kinetic theory explanation for these differences between the Enskog model and the present model is in progress.

\section{$5 \quad$ Efficiency}

The model presented here runs with nearly the same efficiency as standard DSMC at low densities. The calculation of displacements and the use of the $Y$ factor only increase the computational cost by one or two percent. At low densities, HS MD is inefficient because of the large number of possible collision partners within a neighborhood of a few mean free paths [23]. The number of operations per collision per particle with hard sphere dynamics grows as $n^{-2}$ at low densities, while it is independent of density for DSMC. In comparison with a 
scalar hard spheres molecular dynamics code, the dense gas DSMC scheme runs two orders of magnitude faster for $n^{*}=0.01414$. This advantage can be further enhanced by running on a parallel architecture [24].

At high densities, the dense gas DSMC method becomes inefficient compared with HS MD. The reason is that a cell the size of a mean free path, namely one which is roughly $1 / 10$ of a HS diameter represents only a small fraction $(1 / 1000)$ of a single hard sphere particle. Thus 20 million particles are required to represent $1000 \mathrm{HS}$ particles, assuming 20 DSMC particles per cell. On a single processor computer, HS MD and dense gas DSMC are of comparable efficiency at $n^{*} \approx 0.3$, while on a massively parallel machine (with 1000 processors) this "break-even" density increases to $n^{*} \approx 0.7$.

\section{Conclusions}

In this paper a modification of the DSMC algorithm which extends the method to dense gases is described. Computer simulations of this method yielded the equilibrium thermodynamic and nonequilibrium transport properties. In general, for all properties good agreement was found with HS MD at densities less than $n^{*}=0.3$. Further exploration of the effects of time step, spatial grid, effective number, and overall system size is necessary for more quantitative comparisons.

Direct simulation Monte Carlo has been a popular method for the simulation of hydrodynamic flows of high Knudsen number where conventional Navier-Stokes solvers are inaccurate. Since most DSMC applications have been in rarefied flows, the method's restriction to ideal gases has not been viewed as a major drawback. This dense gas version of DSMC will extend the method's utility to a variety of new problems, which involve not only very low density gases, but moderate density as well. These include the study of cold boundary layers in high altitude flows and strong shocks [25].

\section{Acknowledgments}

We thank G. L. Eyink, A. J. C. Ladd, M. Malek Mansour and M. Mareschal for a number of very helpful discussions. This work was carried out under the auspices of the Department of 
Energy at Lawrence Livermore National Laboratory under Contract \#W-7405-ENG-48.

\section{References}

[1] G. A. Bird, Molecular Gas Dynamics and the Direct Simulation of Gas Flows (Clarendon, Oxford, 1994).

[2] A. L. Garcia, Numerical Methods for Physics, Chapter 10 (Prentice Hall, Englewood Cliffs NJ, 1994).

[3] W. Wagner, J. Stat. Phys. 66, 1011 (1992).

[4] M.P. Allen and D.J. Tildesley, Computer Simulation of Liquids, (Clarendon, Oxford, 1987).

[5] Modified collision rates are commonly used in DSMC to reproduce the transport properties of non-hard sphere gases e.g., the Maxwell molecule and Bird's variable hard sphere model [1]. However, these modifications retain the ideal gas equation of state. See also $\mathrm{F}$. Baras, M. Malek Mansour and A.L. Garcia, Phys. Rev. E 493512 (1994).

[6] M. A. Fallavollita, D. Baganoff, and J. D. McDonald J. Comp. Phys. 10930 (1993).

[7] E.P. Muntz, Ann. Rev. Fluid Mech. 21387 (1989).

[8] D. A. Erwin, G. C. Pham-Van-Diep and E. P. Muntz, Phys. Fluids A 3697 (1991).

[9] D. C. Wadsworth, Phys. Fluids A 5, 1831 (1993).

[10] D. L. Morris, L. Hannon and A. L. Garcia, Phys. Rev. A 46, 5279 (1992).

[11] E. Salomons and M. Mareschal, Phys. Rev. Lett. 69, 269 (1992).

[12] M. Malek Mansour, A. L. Garcia, G. C. Lie and E. Clementi, Phys. Rev. Lett. 58, 874 (1987).

[13] S. M. Dunn and J. B. Anderson J. Chem. Phys., 996607 (1993).

[14] M. M. Mansour and F. Baras Physica A, 188253 (1992). 
[15] F. J. Alexander, A. L. Garcia and B. J. Alder Phys. Fluids (to appear) (1994).

[16] Moving to contact, each point particle travels an extra distance $\sigma / 2$ (as compared with hard rods). Moving apart after the collision, each point particle must also travel an additional distance $\sigma / 2$.

[17] P. Resibois and M. De Leener, Classical Kinetic Theory of Fluids, (John Wiley and Sons, New York, 1977).

[18] J. J. Erpenbeck and W. W. Wood, J. Stat. Phys. 35321 (1984); J. J. Erpenbeck and W. W. Wood, J. Stat. Phys. 40787 (1985).

[19] B. J. Berne and R. Pecora Dynamic Light Scattering, Krieger Publ., Malabar, Fla. (1976).

[20] B. J. Alder, D. M. Gass, and T. E. Wainwright, J. Chem. Phys. 533813 (1970).

[21] The relative magnitude of the grid error goes as $(\Delta y / \lambda)^{2}$; this undesirable grid effect may be minimized by using a cell/subcell hierarchy [1].

[22] The cross and potential contributions exclude the grid error (second term in (6)) and only count the post collisional displacement (third term in (6)).

[23] M. Reed and K. Flurchick, Comp. Phys. Comm. 8156 (1994).

[24] M. A. Fallavollita, J. D. McDonald and D. Baganoff, Comp. Sci. Eng. 3283 (1992).

[25] A. Frezzotti and C. Sgarra, J. Stat. Phys 73193 (1993).

\section{Figure Captions}

- Figure 1. Schematic illustration of the displacement occurring after a collision.

- Figure 2. Pressure (normalized by ideal gas pressure) as a function of number density for a time step of $\Delta t=0.04 \lambda /\langle v\rangle$ and the cell width is $\lambda$. The solid line is HS MD.

- Figure 3. Viscosity versus number density as measured using the Einstein relation (circles), transverse current correlation function (diamonds), velocity sine wave decay (tri- 
angles) and Poiseuille flow (squares); the solid line is Enskog theory and the dashed line is HS MD.

- Figure 4. Thermal conductivity versus number density as measured using the Einstein relation (circles); the solid line is Enskog theory and the dashed line is HS MD.

- Figure 5. Velocity versus position in a channel of length $L$ for $n^{*}=0.1414$. The solid line is the quadratic fit of the data. The cell width is $0.38 \lambda$, and the time step is $\Delta t=0.038 \lambda /\langle v\rangle$. 

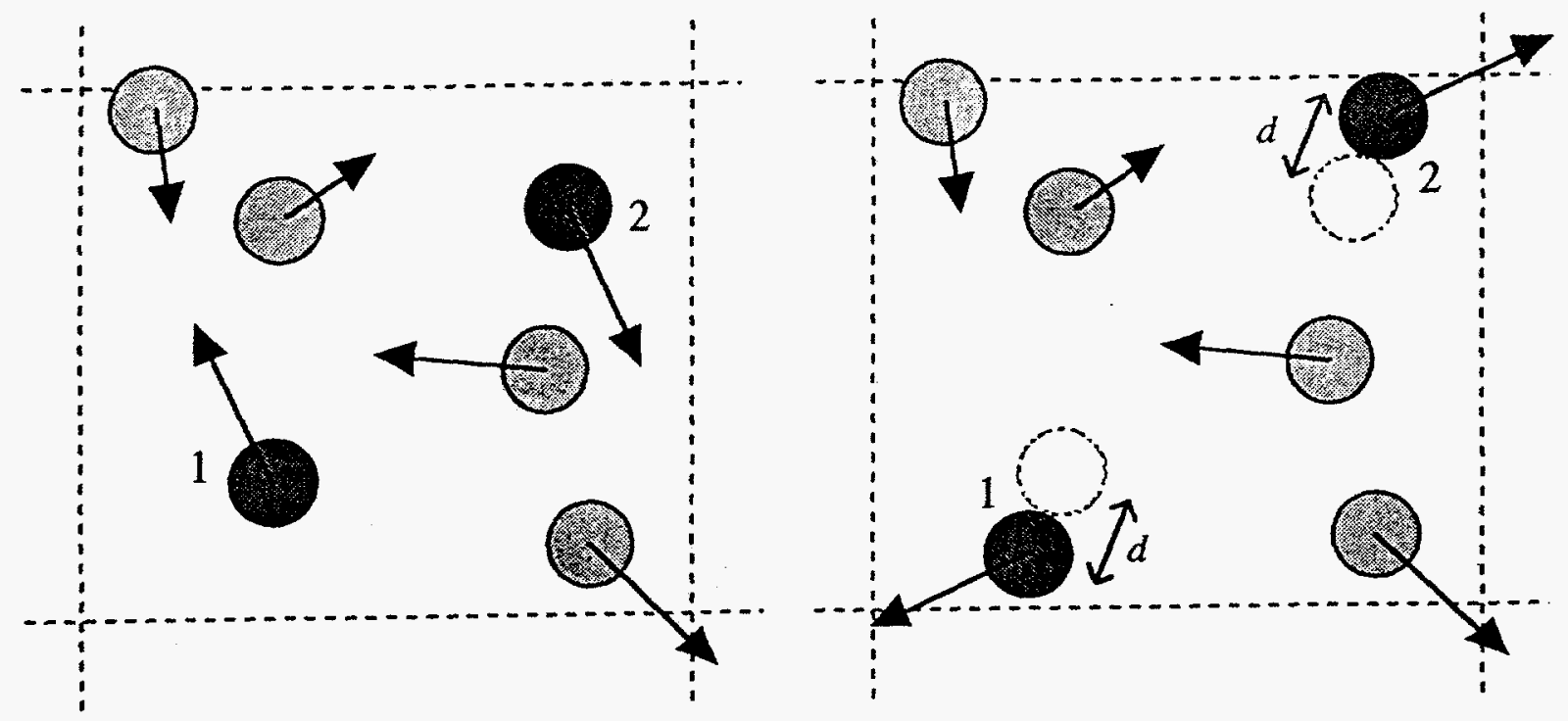

Fig 


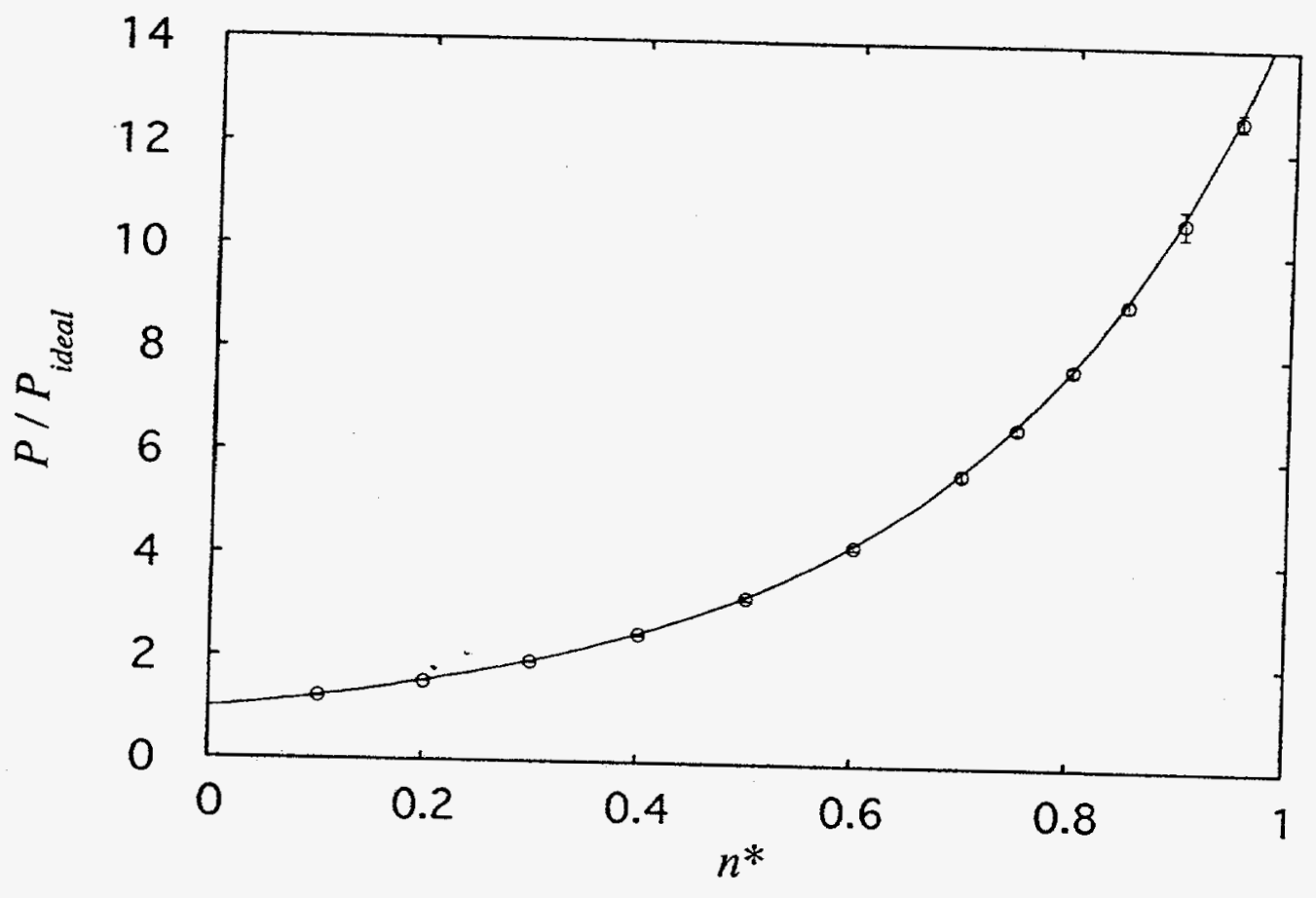

Fig 2 


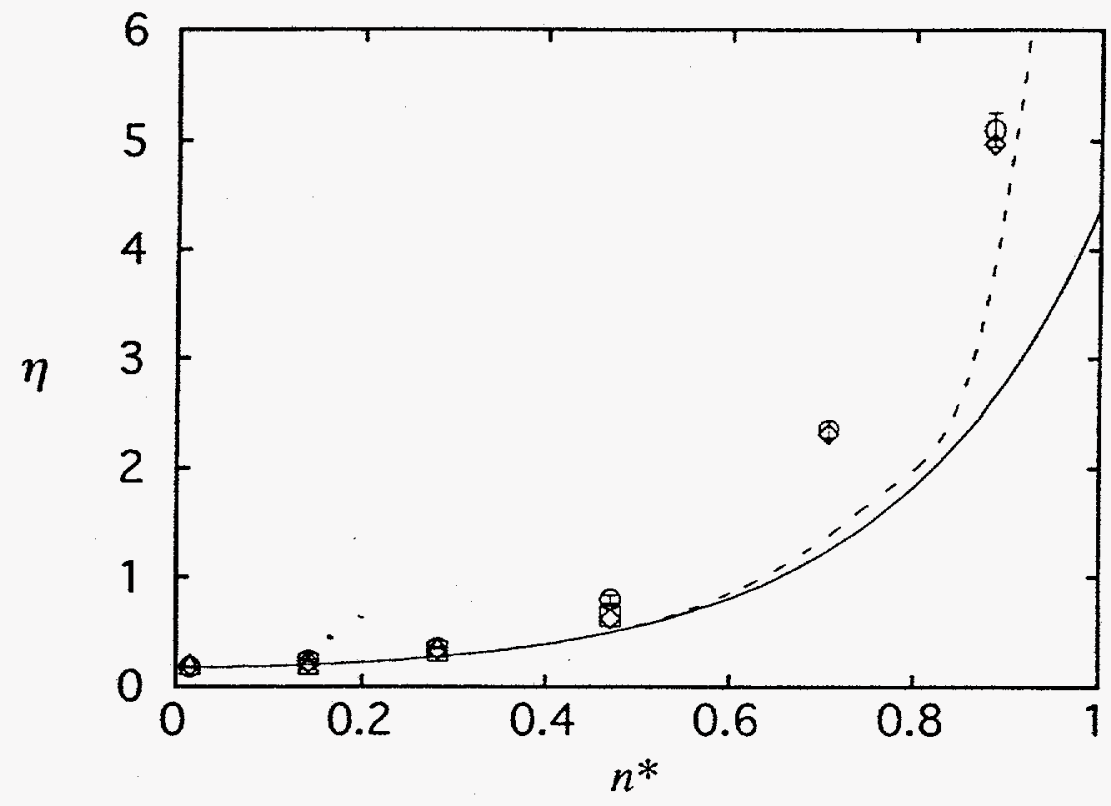

$=-$ 


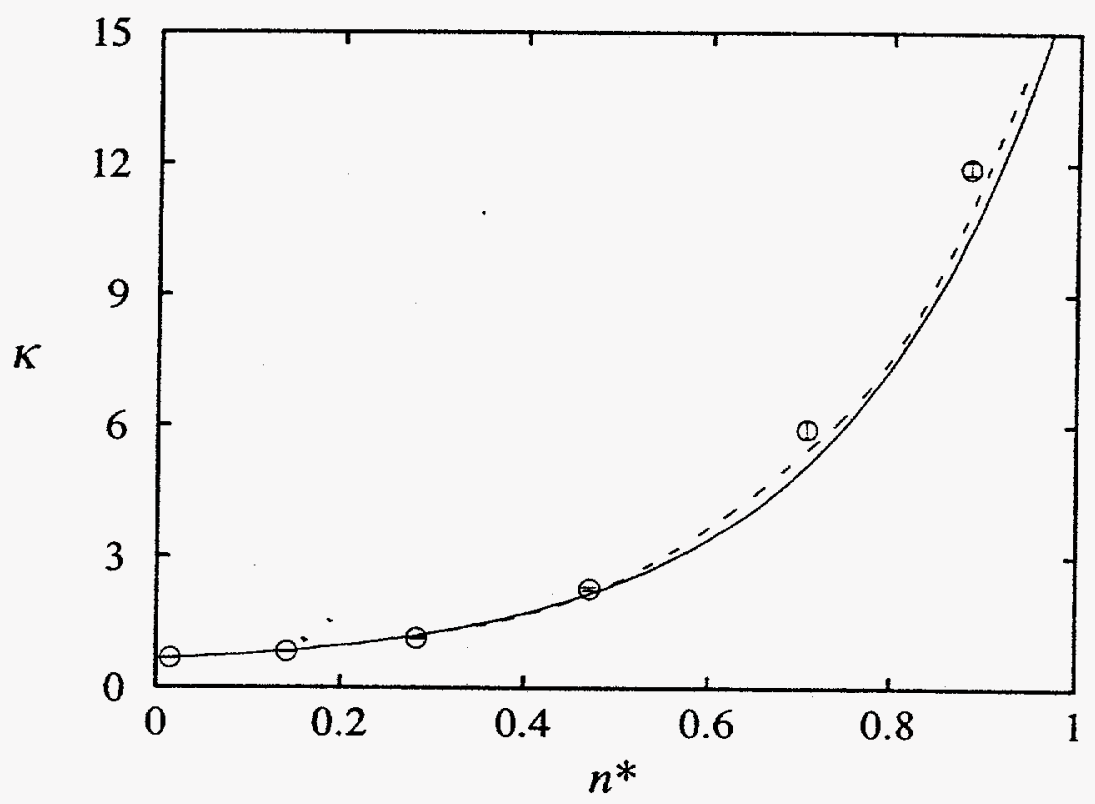

Fia 


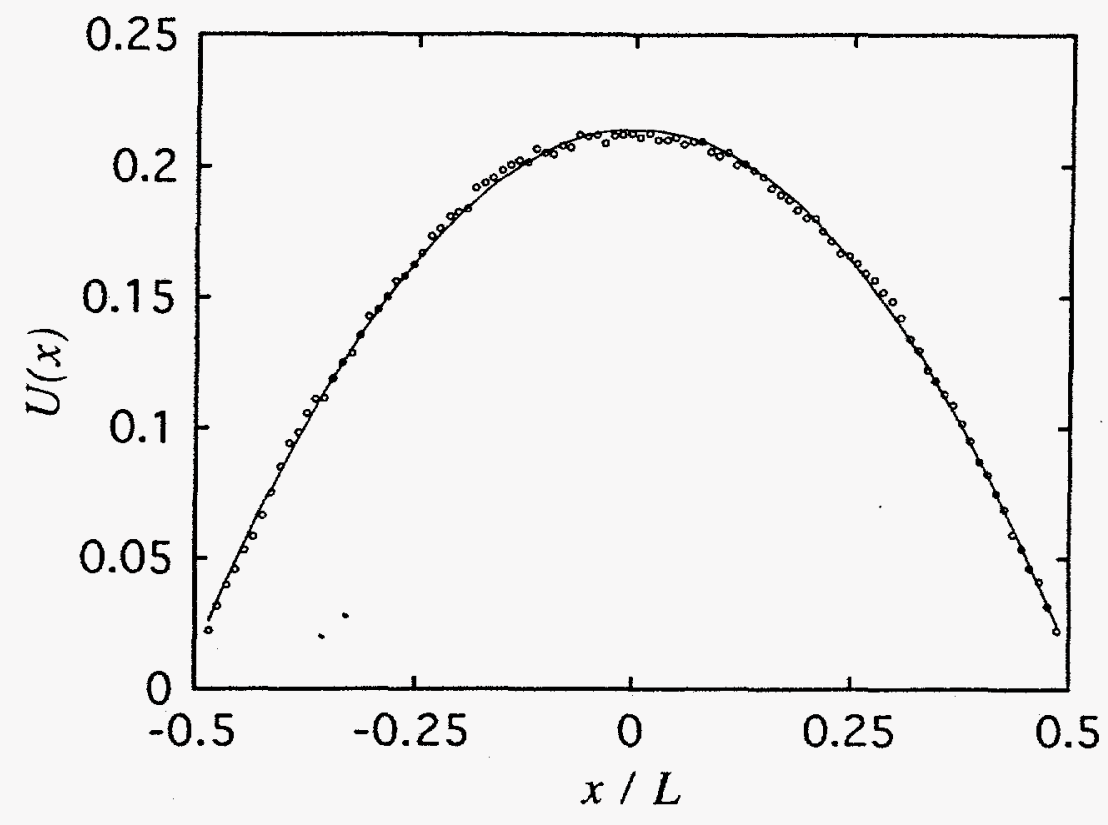

Fig $E$ 